\title{
Italy toasts Sardinia with SharDNA
}

Last month, the Sardinian geneticist Mario Pirastu announced the creation of Italy's first genomics company, SharDNA, which has been set up to facilitate gene discovery among Sardinia's population. Whereas some scientists argue that many isolated populations offer no benefit over general populations for genetic studies, others point out that Sardinia has some specific advantages. Creation of the company marks the first time one of Italy's major public agencies has collaborated with industry, and is an attempt by Sardinia to take charge of exploitation of its own genetic resources.

Based in Cagliari, SharDNA is the product of a collaboration between Pirastu and Italy's most successful entrepreneur, Renato Soru, who among other things launched Italy's first free internet provider, Tiscali, which has just acquired $56 \%$ of World Online, making it the 2nd European internet provider with operations in 15 countries. The creation of SharDNA marks Soru's first investment in biotechnology - a move that is thought to have persuaded the National Research Council (Consiglio Nazionale delle Ricerche, $\mathrm{CNR}$ ) to become involved with a private company for the first time: Soru has invested an initial $\$ 3.5$ million for a $75 \%$ share in the company, the remaining $25 \%$ being held by two Sardinian banks (Banco di Sardegna and Sfirs), a private clinic, and the CNR.

According to Pirastu, who heads the CNR molecular genetics institute in Alghero, SharDNA (named from Shardana, the island's old name) will collaborate on research projects in the Ogliastra genetic park, providing funds to study Sardinian genetic inheritance. The Ogliastra genetic park is a public consortium created 8 months ago to study genetic epidemiology in several villages located in the centre of Sardinia. So far only 2 villages, Talana and Perdasdefogu, are involved but the park hopes to extend to 10 genetic isolates. SharDNA is currently looking to collaborate with nonItalian biotechnology or pharmaceutical companies interested in exploiting genetic data collected in Sardinian villages/isolates.

Several other genetically isolated populations have attracted the interest of genomics companies in recent years. For instance, DeCODE Genetics (Reykjavik) is searching for disease genes among Iceland's 270,000 citizens (Nat. Biotechnol. 16, 896), Gemini Genomics (Cambridge, UK) is looking at the population in Newfoundland (Nat. Biotechnol. 18, 366), and and the Umeå University in Sweden has created a company to study the 60,000 Sami living in the Västerbotten county. But some

Anna Meldolesi is a freelance writer working in Rome. geneticists now argue that some homogeneous populations-particularly those stemming from many founders-are not as valuable as once thought for untangling multiple factors involved in complex disease. While some argue that association studies are limited by small population size, others, such as John Todd, professor of medical genetics at the Cambridge institute for medical research, show that the likelihood of a gene marker pinpointing a gene in these populations is not always high (Nat. Genet. 25, 320323, 2000). As a result, there is growing support for the more expensive and time-consuming approach of whole genome studies of large heterogeneous populations projects. Such projects include the UK's Medical Research Council and Wellcome Trust plan to look for cardiovascular disease variables in 500,000 of the general population.

However, James Weber of the US National Institutes of Health (NIH; Bethesda, MD), which as part of the consortium is funding a genome polymorphism screen in the villages with a special interest in mapping genes influencing kidney stones, hypertension, and asthma, points out that not all homogenous populations are equal. Unlike those with many founders-such as in Iceland, Finland, and Sardinia (if you consider the entire population)_communities in the Ogliastra villages exhibit high linkage disequilibrium, meaning that 2 alleles or gene variants tend to stay together down the generations, thereby increasing the likelihood that a gene marker will pinpoint a gene. For example, Talana, which currently comprises 1,200 inhabitants, was established a thousand years ago by an extremely small number of original settlers$75 \%$ of its population descends from 8 founder fathers and 10 founder mothers. $95 \%$ of the community results from marriages within the same village, and $35 \%$ of marriages are consanguineous. "Talana with its few founders seems to be a nearly ideal case for gene discovery," says Weber. So while sampling Sardinia's entire population may not be beneficial, looking specifically in the villages with few founders and comparing results will be advantageous.

In addition, genealogical data in several Ogliastra villages are very good, points out Alan Wright of Edinburgh's Medical Research Council, which is a member of the Ogliastra genetic park. "In Talana, families can be traced back to the $17^{\text {th }}$ century using church records-this is another pro," he says.

Its million and half inhabitants have long been the target of genetics and population genetics studies and so, as Soru explains, there is much riding on SharDNA's success: "For a long time, geographic marginality has been a handicap and Sardinia has been a game reserve for people from outside," he says. "Searching for genes from within Sardinia's isolated communities is a way to turn things around to our advantage."

Anna Meldolesi

\section{PERV data renew xeno debate}

Two pieces of news in August revived the debate about whether the technical hurdle of preventing transmission of porcine endogenous retrovirus (PERV) during xenotranplantation can be overcome. In mid-August, a team from the Scripps Research Institute (La Jolla, CA) announced what they claim is the first evidence of cross species PERV infection. Then two weeks later, at the $18^{\text {th }}$ International Congress of the Transplantation Society in Rome, BioTransplant (Boston, MA), a biotechnology company focused on transplant tolerance, presented data indicating that it may have developed a breed of pig that doesn't produce PERV capable of infecting human cells. However, neither piece of news is definitive, and investors perceptions of xenotransplantaion safety remain unchanged.

The Scripps work seems to show crossspecies transmission of PERV from pig cells to mice. Two months after transplanting pig pancreatic islets into immunodeficient mice, the islets continued to make infectious virus particles, according to one of the authors Daniel
Salomon, associate professor at the Scripps department of molecular and experimental medicine. In addition, some mouse tissues in non-transplant sites like the liver and spleen were found to be infected. The group also showed PERV can infect isolated human cells in vitro (Nature, 407, 501-504, 2000). "If you transplant pig tissues - at least as pig tissues exist today-there's a real possibility that you're going to move PERV. . .from these pig tissue into humans," concludes Salomon.

However, the results do not convince all observers. Jay Fishman, clinical director, transplant infectious diseases, Massachusetts General Hospital and a consultant to BioTransplant and various pharmaceutical companies, points out that detection of infection is not clearcut. Limits on technology available at present mean "we can't tell directly whether or not there is infection by PERV of host tissue when there are pig cells floating around," he says. "We impute the fact that there is infection based on a variety of ratios of PCR signals."

Alan Colman, director of worldwide 